\title{
Realtime autonomous navigation in V-Rep based static and dynamic environment using EKF-SLAM
}

\author{
Umme Hani, Lubna Moin \\ Department of Electrical Engineering, National University of Sciences \& Technology, Karachi, Pakistan
}

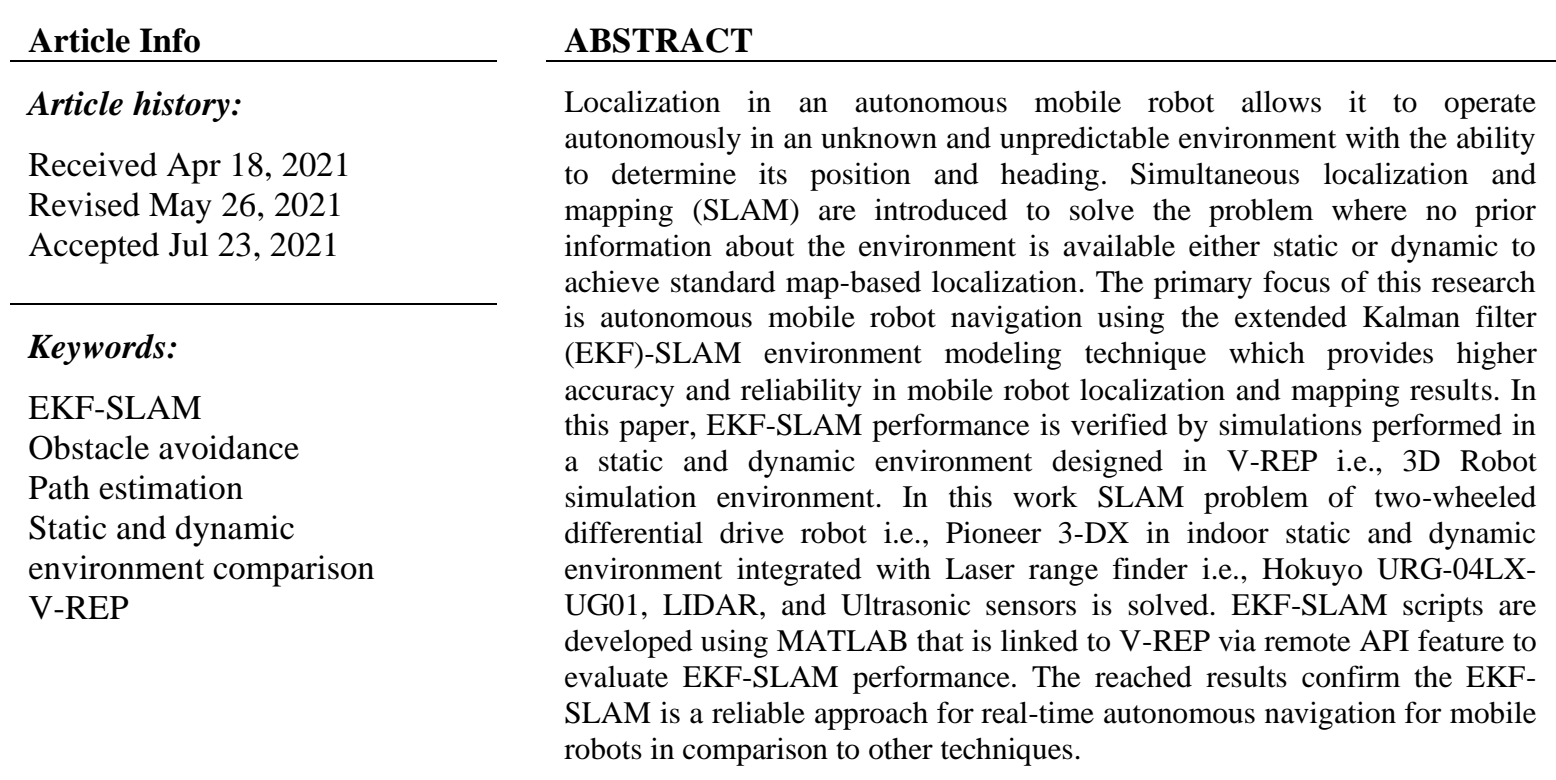

This is an open access article under the CC BY-SA license.

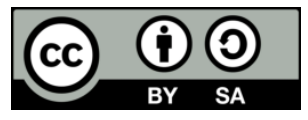

\section{Corresponding Author:}

Umme Hani

Department of Electrical Engineering

National University of Sciences and Technology

PNEC Road, PNS Jauhar Karsaz Faisal Cantonment, Karachi, Pakistan

Email: umme.hani2017@pnec.nust.edu.pk,ummehani098@gmail.com

\section{INTRODUCTION}

Autonomous mobile robots' navigation is considerably a matter of contention for the last two decades. researchers and scientists are putting prodigious efforts to find reliable solution with the help of artificial intelligence and computer vision. The applications of mobile robots are proficient with a degree of self-sufficiency and the criteria of its autonomy are sensing the environment, self-maintenance, task performing, autonomous navigation that includes indoor and outdoor navigation while in cluttered environments reliable data association is important [1] because wrong data association can have catastrophic consequences.

Current simultaneous localization and mapping (SLAM) approaches include the graph-SLAM method, particle filtering method (PF), scan matching method (SM), and the extended Kalman filter method (EKF). The graph-SLAM method takes the SLAM problem as an optimization problem [2]. The objective function and constraints are defined, and it is solved using the mathematical programming method. The PF method is a recursive Bayesian estimator for dynamic Bayesian networks that approximates the PDF i.e. 
probability density function by searching for the cluster of random samples, uses the sample mean to replace the integral operations, and acquires the variance distribution of the state [3]. It has a special advantage in dealing with status filtering of parameter estimation of non-Gaussian and nonlinear time-varying systems while the major drawback is in high-dimensional spaces sampling that can be inefficient. The SM method minimizes the metric distances between the scan characteristics or original data, and aligns the overlap of the scanning datasets [4]. The SLAM process leads to better navigation in dynamic environments autonomously. However, a lack of proprioceptive feedback from an autonomous mobile robot may cause trouble in the autonomous operation of the robot in unpredictable and unknown environments. Above mentioned issues are some basic problems of SLAM. Nowadays, many researchers analyze different approaches to solving problems in SLAM and it is analyzed that the approach based on EKF among all approaches are the most commonly used to solve SLAM problems. Teslić et al. [5] uses LabMate ${ }^{\mathrm{TM}}$.to show robot navigation in indoor environment with integrated use of laser rangefinder sensor.

EKF-SLAM is one of the probabilistic approaches to control uncertainties of any mobile robot [6]-[8] is most widely used in mobile robotics, especially in SLAM. Moreover, the SLAM filtering solution, which is based on the EKF application is the first successfully implemented [9], [10], and most often used online SLAM algorithm. There are many theoretical and practical works dedicated to EKF usage with different approaches [11], [12], and application fields [13], [14]. In the autonomous robot navigation, the EKF based SLAM is categorized as non-linear SLAM, where linearization of non-linear models along with a summation of system noise with Gaussian filter takes place so that the Kalman filter algorithm can be applied. In comparison with the Kalman filter, EKF-SLAM represents the non-linear models which are an essential part of all navigation problems almost. EKF-SLAM estimates mobile robot location as it is moving using incremental maximum likelihood estimator, it generates the map by localization and generated map information uses to update its current states and map simultaneously [15].

In this work, the EKF-SLAM algorithm has been successfully implemented using a pioneer 3-DX i.e., a small lightweight two-wheel differential drives mobile robot. The algorithm is implemented and its performance is analyzed in a clustered environment created on V-REP. Lidar sensor interfaced with robot model and the output of robot motors, sensors are then integrated with MATLAB to apply the algorithm and observe its performance in a static and dynamic environment.

\section{RESEARCH METHOD}

The mobile robot model predicts the current states based on control input and previous states of the robot is shown in Figure 1. Mathematically, the process model in the discrete form can be written as (1). The robot position $(\mathrm{x}, \mathrm{y})$ and orientation angle i.e., $\Theta$ estimated the robot states in this research work. It can be shown in the form of a state vector as (2). In this research work speed and angular velocity is applied as control input can be exhibit as in (3).

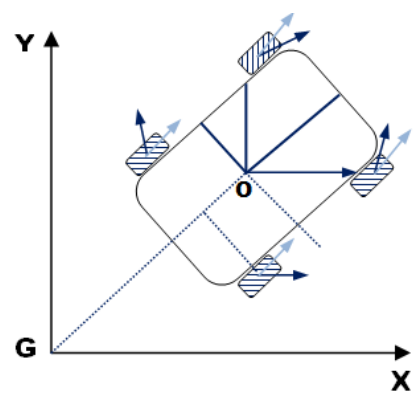

Figure 1. Robot model

$$
\begin{aligned}
& R_{(n+1)}=f\left(R_{(n)}, u_{(n)}\right) \\
& R=\left[\begin{array}{l}
\mathrm{x} \\
\mathrm{y} \\
\theta
\end{array}\right] \\
& u_{(n)}=\left[\begin{array}{l}
\mathrm{v}_{(\mathrm{n})} \\
\omega_{(\mathrm{n})}
\end{array}\right]
\end{aligned}
$$


In discrete-time, iteratively for every $\mathrm{n}^{\text {th }}$ sample, the predictive state of the robot is predicted in sample time T, which capture in time (n. T).

Since the control input to the robot models are speed and angular velocity that is measured and used to predict robot states in this process. The discrete-time robot kinematics model for the above case is expressed in (4).

$$
R_{(n+1)}=\left[\begin{array}{c}
x_{(n+1)} \\
y_{(n+1)} \\
\theta_{(n+1)}
\end{array}\right]\left[\begin{array}{l}
x_{(n)} \\
y_{(n)} \\
\theta_{(n)}
\end{array}\right]+T\left[\begin{array}{c}
v_{(n)} \cdot \cos \left(\theta_{(n)}\right) \\
v_{(n)} \cdot \sin \left(\theta_{(n)}\right) \\
\omega_{(n)}
\end{array}\right]
$$

Robot model Jacobian can be represented in (5).

$$
R_{(n+1) \mid n}=R_{(n) \mid n}+T\left[\begin{array}{c}
v_{(n)} \cdot \cos \left(R(3)_{(n) \mid n}\right) \\
v_{(n)} \cdot \sin \left(R(3)_{(n) \mid n}\right) \\
\omega_{(n)}
\end{array}\right]
$$

Ideally, the current states of the robot can be estimated accurately by using the above robot kinematics model but in practice, it is influenced by errors that can be noise in the measurement of sensors and friction. Equation (1) with the addition of the noise model can be written as (6).

$$
R_{(n+1)}=f\left(R_{(n)}, u_{m(n)}+\delta \mathrm{u}_{(\mathrm{n})}\right)=f\left(R_{(n)}, u_{m(n)}\right)+\delta f
$$

In the above case, the noise model is estimated as Gaussian noise with zero mean represented by $\delta f$. The covariance of this system noise originated during the measurement process can be calculated as it can be seen in (7).

$$
Q f=F_{u} \cdot Q_{u} \cdot F_{u}^{T}
$$

In which:

$Q f=$ Covariance of Process noise

$Q u=$ Covariance of Control input measurement

$F u=\delta_{f} \delta_{(v, \omega)}$

The process model Jacobian matrices concerning the control input measurement $F_{u}$ and robot states $F_{\mathrm{R} v}$ can be expressed in (8) and (9), respectively.

$$
\begin{aligned}
& \mathrm{F}_{\mathrm{u}}=\frac{\delta \mathrm{f}}{\delta(\mathrm{x}, \mathrm{y}, \theta)}=\left[\begin{array}{ccc}
1 & 0 & -\mathrm{T} \cdot \mathrm{v}_{(\mathrm{n})} \cdot \sin \left(\theta_{(\mathrm{n})}\right) \\
0 & 1 & \mathrm{~T} \cdot \mathrm{v}_{(\mathrm{n})} \cdot \cos \left(\theta_{(\mathrm{n})}\right) \\
0 & 0 & 1
\end{array}\right] \\
& \mathrm{F}_{\mathrm{u}}=\frac{\delta \mathrm{f}}{\delta(\mathrm{v}, \mathrm{\omega})}=\left[\begin{array}{cc}
\mathrm{T} \cdot \cos \left(\mathrm{R}(3)_{(\mathrm{n})}\right) & 0 \\
\mathrm{~T} \cdot \sin \left(\mathrm{R}(3)_{(\mathrm{n})}\right) & 0 \\
0 & \mathrm{~T}
\end{array}\right]
\end{aligned}
$$

\subsection{SLAM operation based on 2D-EKF}

The SLAM process flow in this research work can be observed in Figure 2. In contrast to EKF localization SLAM operation involves the initialization of landmarks to update robot position.

The states during the process that are estimated consist of estimated landmarks and robot states. Point landmarks are used in this research, which has two-dimensional (x and y) states. Equation (10) exhibits the SLAM operation based whole estimated states vector. The mobile robot states $(\mathrm{x}, \mathrm{y}, \theta)$ are correlated by $\mathrm{R}_{\mathrm{V}}$ and set of landmark states $\left(\mathrm{L}_{\mathrm{x}_{1}}, \mathrm{~L}_{\mathrm{y}_{1}}, \mathrm{~L}_{\mathrm{x}_{2}}, \mathrm{~L}_{\mathrm{y}_{2}}, \ldots, \mathrm{L}_{\mathrm{x}_{\mathrm{n}}}, \mathrm{L}_{\mathrm{y}_{\mathrm{n}}}\right)$ are correlated by $\mathrm{R}_{1}$, with $\mathrm{n}$ is the registered landmark number. Similar to the localization process, in which EKF is used to estimate the states and Gaussian variables including covariance matrix $P$ and mean i.e., the expected value of state vector is used to model all the processes. The correspondent of the expected value of the state vector and its covariance matrix $\mathrm{P}$ can be shown in (11) and (12). These two matrices expand every time in size with the initialization of the landmark process, whenever the mobile robot detects a new landmark. 


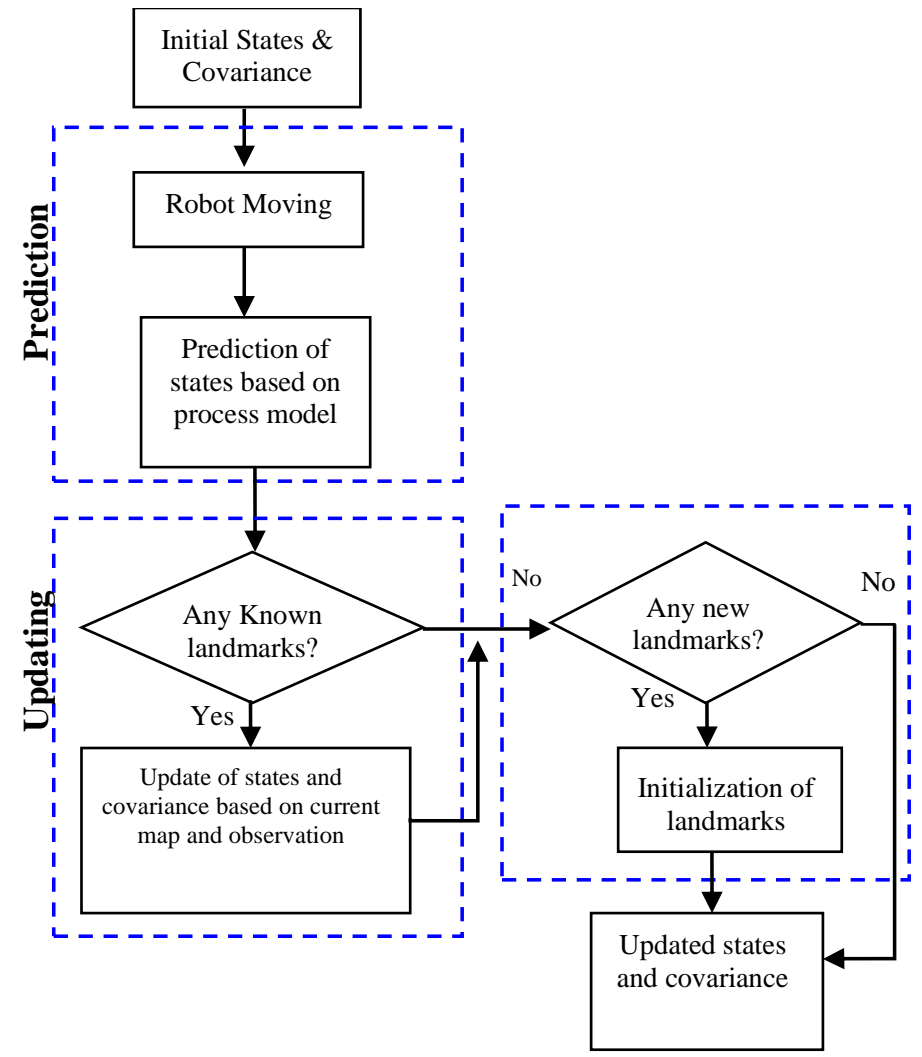

Figure 2. 2D EKF-SLAM operation

$$
\begin{aligned}
& \mathrm{R}=\left[\begin{array}{l}
\mathrm{R}_{\mathrm{V}} \\
\mathrm{R}_{1}
\end{array}\right]=\left[\begin{array}{c}
\mathrm{x} \\
\mathrm{y} \\
\theta \\
\mathrm{L}_{\mathrm{x}_{1}} \\
\mathrm{~L}_{\mathrm{y}_{1}} \\
\vdots \\
\mathrm{L}_{\mathrm{x}_{\mathrm{n}}} \\
\mathrm{L}_{\mathrm{y}_{\mathrm{n}}}
\end{array}\right] \\
& \mathrm{R}=\left[\begin{array}{l}
\overline{\mathrm{R}_{\mathrm{V}}} \\
\overline{\mathrm{R}_{1}}
\end{array}\right]=\left[\begin{array}{c}
\overline{\mathrm{R}_{\mathrm{V}}} \\
\overline{\mathrm{L}_{1}} \\
\cdot \\
\overline{\overline{\mathrm{L}}_{1}}
\end{array}\right] \\
& \mathrm{P}=\left[\begin{array}{ll}
\mathrm{P}_{\mathrm{X}_{\mathrm{V}}, \mathrm{X}_{\mathrm{V}}} & \mathrm{P}_{\mathrm{X}_{\mathrm{V}}, \mathrm{X}_{1}} \\
\mathrm{P}_{\mathrm{X}_{1}, \mathrm{X}_{\mathrm{V}}} & \mathrm{P}_{\mathrm{M}, \mathrm{X}_{1}}
\end{array}\right] \\
& \mathrm{P}=\left[\begin{array}{ccccc}
\mathrm{P}_{\mathrm{X}_{\mathrm{V}}, \mathrm{X}_{\mathrm{V}}} & \mathrm{P}_{\mathrm{X}_{\mathrm{V}}, \mathrm{L}_{1}} & \cdot & \cdot & \mathrm{P}_{\mathrm{X}_{\mathrm{V}}, \mathrm{L}_{\mathrm{n}}} \\
\mathrm{P}_{\mathrm{L}_{1}, \mathrm{X}_{\mathrm{V}}} & \mathrm{P}_{\mathrm{L}_{1}, \mathrm{~L}_{1}} & \cdot & \cdot & \mathrm{P}_{\mathrm{L}_{1}, \mathrm{~L}_{\mathrm{n}}} \\
\vdots & : & & & \vdots \\
\mathrm{P}_{\mathrm{L}_{1}, \mathrm{X}_{\mathrm{V}}} & \mathrm{P}_{\mathrm{L}_{1}, \mathrm{X}_{\mathrm{V}}} & \cdot & . & \mathrm{P}_{\mathrm{L}_{n}, \mathrm{~L}_{n}}
\end{array}\right]
\end{aligned}
$$

\subsection{D EKF-based SLAM process general operation}

\subsubsection{Step 0. robot initialization}

At the initial time ( $\mathrm{t}=0)$, only the robot states are initial states, and no landmark is registered to the map. Although, $\mathrm{P}=\mathrm{P} 0$ can be considered as the covariance matrix as the robot initial state is assumed to be known so that there is no uncertainty. 
$\overline{\mathrm{R}}=\overline{\mathrm{R}_{\mathrm{V}}}=\left[\begin{array}{l}\mathrm{X} \\ \mathrm{y} \\ \theta\end{array}\right]=\left[\begin{array}{l}0 \\ 0 \\ 0\end{array}\right] \mathrm{P}=\left[\begin{array}{lll}0 & 0 & 0 \\ 0 & 0 & 0 \\ 0 & 0 & 0\end{array}\right]$

\subsubsection{Step 1. prediction step}

\section{a. Updating robot states}

The mobile robot movement affects the robot states only and based on the robot process model and its movement, the new estimated robot state $\left(R_{V}\right)$ as in $(15)$ and the landmark state $\left(R_{1}\right)$ as in $(16)$ can be predicted.

$$
\begin{aligned}
& \overline{\mathrm{R}_{\mathrm{V}}} \leftarrow \mathrm{f}_{\mathrm{R}_{\mathrm{v}}}\left(\overline{\mathrm{R}_{\mathrm{V}}}, \mathrm{u}, \overline{\mathrm{N}}\right) \\
& \overline{\mathrm{R}_{1}} \leftarrow \overline{\mathrm{R}_{1}}
\end{aligned}
$$

Equation (15) correlates to the robot process model (4) based on its control input (u), previous state $\left(\mathrm{R}_{\mathrm{V}}\right)$ and noise model $(\mathrm{N})$ while $\overline{\mathrm{N}}$ is equal to zero as the noise in this process is modeled in as white noise.

\section{b. Robot covariance updating}

The updated covariance $\mathrm{P}$ in this process based on model prediction is calculated as in (17), where corresponding to the process model equation, $\mathrm{F}_{\mathrm{R}}$ is the Jacobian of the state and $\mathrm{Pn}$ is the measurement input control noise covariance.

$$
\mathrm{P} \leftarrow \mathrm{F}_{\mathrm{R}} \mathrm{PF}_{\mathrm{R}}^{\mathrm{T}}+\mathrm{Pn}
$$

As mentioned previously, states of the robot $(\bar{R})$ are only affected by the robot movement so the covariance matrix $\mathrm{P}$ related to the robot states affects the Jacobian matrix to update. Therefore, in this process, the Jacobian matrix is calculated as in (18), in which 0 corresponds to zero matrices and I correspond to the identity matrix.

$$
F_{x}=\left[\begin{array}{cc}
\frac{d f_{R}}{d R} & 0 \\
0 & I
\end{array}\right] P n=\left[\begin{array}{c}
\frac{d f_{R}}{d N} \\
0
\end{array}\right]
$$

\subsubsection{Step 2. landmark-based observation updating process}

The mobile robot observes around the landmark while it is moving using the laser sensor that measures observable landmarks range and bearing related to the robot orientation and position. If the observed landmark is already registered to the map, its range and bearing measurement are used to update the state's estimation $(\overline{\mathrm{R}})$ and also its covariance $(\mathrm{P})$. The measurement process and its corresponded covariance modeled as in (19) and (20) are independent for each landmark (i). The state updating process based on observed landmarks is processed one by one of each landmark.

$$
\begin{aligned}
& \overline{\mathrm{z}}=\mathrm{y}_{\mathrm{i}}-\mathrm{h}_{\mathrm{i}}\left(\mathrm{R}_{\mathrm{V}}, \mathrm{L}_{\mathrm{i}}\right) \\
& \mathrm{H}_{\mathrm{R}}=\left[\begin{array}{llllllll}
\mathrm{H}_{\mathrm{R}_{\mathrm{v}}} & 0 & \ldots & 0 & \mathrm{H}_{\mathrm{L}_{\mathrm{i}}} & 0 & \ldots & 0
\end{array}\right] \\
& \mathrm{H}_{\mathrm{R}_{\mathrm{V}}}=\frac{\delta \mathrm{h}_{\mathrm{i}}\left(\overline{\mathrm{R}_{\mathrm{V}}}, \overline{\mathrm{L}_{1}}\right)}{\delta \mathrm{R}_{\mathrm{V}}} ; \mathrm{H}_{\mathrm{L}_{\mathrm{i}}}=\frac{\delta \mathrm{h}_{\mathrm{i}}\left(\overline{\mathrm{R}_{\mathrm{V}}}, \overline{\mathrm{L}_{1}}\right)}{\delta \mathrm{L}_{\mathrm{i}}}
\end{aligned}
$$

Based on the Jacobian, see (20) and above measurement model, the updated state process and its updated covariance based on a set of (22), (23), (24), (25), and (26) are calculated, in which $\mathrm{K}$ in this updating process is Kalman gain.

$$
\begin{aligned}
& \overline{\mathrm{z}}=\mathrm{y}_{\mathrm{i}}-\mathrm{h}_{\mathrm{i}}\left(\mathrm{R}_{\mathrm{V}}, \mathrm{L}_{\mathrm{i}}\right) \\
& \mathrm{Z}=\mathrm{H}_{\mathrm{X}} \mathrm{PH}_{\mathrm{X}}^{\mathrm{T}}+\mathrm{R} \\
& \mathrm{K}=\mathrm{PH}_{\mathrm{X}}^{\mathrm{T}} \mathrm{Z}^{-1} \\
& \overline{\mathrm{R}} \leftarrow \overline{\mathrm{R}}-\mathrm{K} \overline{\mathrm{z}} \\
& \mathrm{P} \leftarrow \mathrm{P}-\mathrm{KZK}^{\mathrm{T}}
\end{aligned}
$$




\subsubsection{Step 3. Initialization of landmarks}

When the landmarks for the first time are observed by the robots have not registered on the map. Whereas, based on its range and bearing measurement the state of this new landmark is estimated including $\mathrm{x}$ and y global coordinate corresponding to the robot state R. The new landmark estimated state's function can be exhibit as (27) are calculated as the invert of observation function $\left(\mathrm{h}_{\mathrm{i}}\left(\mathrm{R}, \mathrm{L}_{\mathrm{i}}\right)\right.$ ) while the new landmark states corresponding Jacobian, the inverse observation function and the robot states are written as (28).

$$
\begin{aligned}
& \overline{\mathrm{L}}_{\mathrm{n}+1}=\mathrm{g}\left(\overline{\mathrm{X}}_{\mathrm{V}}, \overline{\mathrm{Y}}_{\mathrm{n}+1}\right) \\
& \mathrm{G}_{\mathrm{X}_{\mathrm{V}}}=\frac{\partial \mathrm{g}\left(\overline{\mathrm{X}}_{\mathrm{V}}, \overline{\mathrm{y}}_{\mathrm{n}+1}\right)}{\partial \mathrm{X}_{\mathrm{V}}} ; \mathrm{G}_{\mathrm{L}_{\mathrm{i}+1}}=\frac{\partial \mathrm{g}\left(\overline{\mathrm{X}}_{\mathrm{V}}, \overline{\mathrm{y}}_{\mathrm{n}+1}\right)}{\partial \mathrm{L}_{\mathrm{i}+1}}
\end{aligned}
$$

The new landmark covariance and cross-covariance are calculated based on (29) and (30) related to the prior states.

$$
\begin{aligned}
& P_{L L}=G_{X_{V}} P_{L L} G_{X_{v}}{ }^{T}+G_{y_{n+1}} X_{v} G_{y_{n+1}}{ }^{T} \\
& P_{L X}=G_{X_{V}} P_{L L} P_{X_{V} X_{1}}
\end{aligned}
$$

Based on the estimation result, these new landmark states and their covariance are then summed into the robot full state, map, and covariance as in (31).

$$
\overline{\mathrm{X}} \leftarrow\left[\frac{\overline{\mathrm{X}}}{\mathrm{L}_{\mathrm{n}+1}}\right] \mathrm{P} \leftarrow\left[\begin{array}{cc}
\mathrm{P} & \mathrm{P}_{\mathrm{LX}}^{\mathrm{T}} \\
\mathrm{P}_{\mathrm{LX}} & \mathrm{P}_{\mathrm{LL}}
\end{array}\right]
$$

\section{RESULTS AND DISCUSSION}

To analyze the performance of EKF-SLAM algorithm for autonomous mobile robot navigation realization, indoor environment with static and dynamic objects was designed on V-REP simulator that was connected with MATLAB via remote API features while two-wheeled differential drive robot i.e., Pioneer 3DX integrated with laser range finder i.e., Hokuyo URG-04LX-UG01 to determine its performance effectiveness with the integration of LIDAR and ultrasonic sensors. EKF- SLAM algorithm is developed using MATLAB that is linked to V-REP via remote API feature. To evaluate EKF-SLAM performance. Figure 3 shows an environment with static objects designed on V-REP and Figure 4 shows an environment with dynamic objects designed on V-REP.

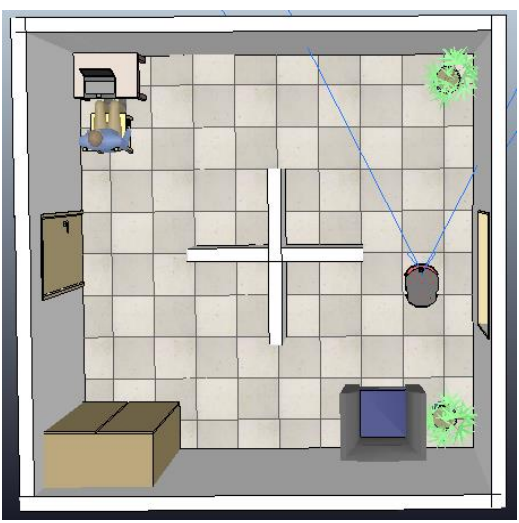

Figure 3. The environment with static objects designed on V-REP

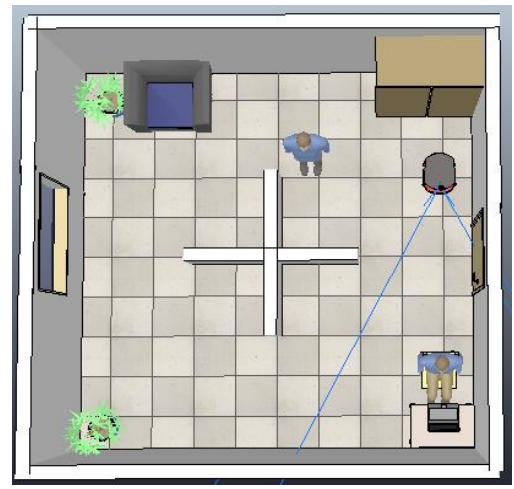

Figure 4. The environment with dynamic objects designed on V-REP

The simulation was performed on V-REP simulator settings i.e., simulation time step $\mathrm{dt}=50 \mathrm{~ms}$, dynamics engine $=$ bullet 2.78 , dynamic settings=accurate. When the simulation begins mobile robot starts scanning with the 2D laser range finder "Hokuyo URG-04LX-UG01" that allows a wide scanning range of $5600 \mathrm{~mm} \times 240^{\circ}$ for measurement of the landmarks in the indoor environment that integrated into the robot 
and avoid obstacles with the help of EKF- SLAM algorithm. In an experiment, the exploration is simulated in a room bounded by $240 \mathrm{~cm}$ high walls, $80 \mathrm{~cm}$ walls in the center, chair, table, cupboard, and rack by a Pioneer 3-DX robot equipped with a mid-noise and odometry 2D range scanner i.e., Hokuyo URG-04LXUG01. Gaussian noise distributed in polar coordinates. Iterative closet point (ICP) algorithm is used for data association [16] i.e., performed at each step and exploration performed with Braintenberg, Lyapunov, ZigZag, and cornering algorithms [17] which maximize performance. It has been observed that uncertainty is the critical measurement parameter of performance degradation which affects measurement models and motion due to its direct influence consistency. Figure 5 shows scanning with static objects on the V-REP simulator and Figure 6 shows scanning with dynamic objects on the V-REP simulator.

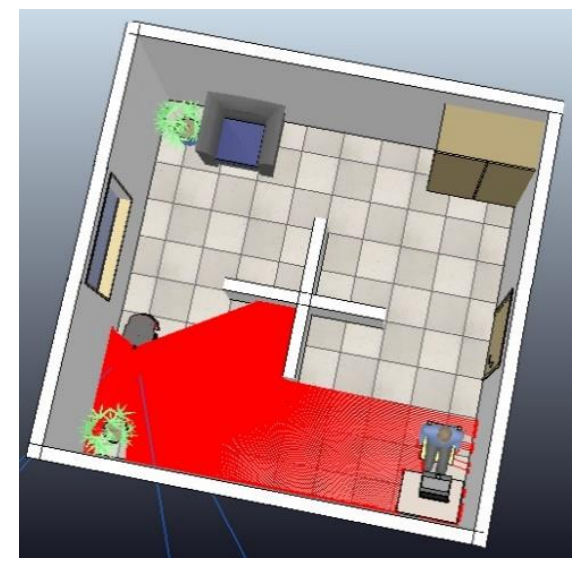

Figure 5. Scanning with static objects on V-REP simulator

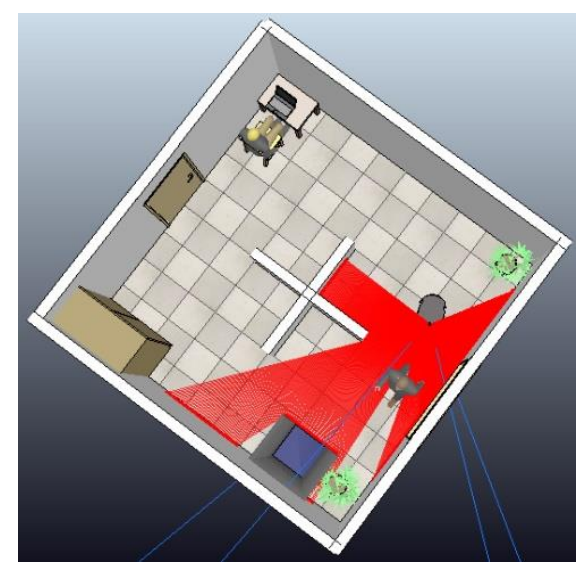

Figure 6. Scanning with dynamic objects on V-REP simulator

Graphical user interface (GUI) designed on MATLAB which was interconnected with V-REP simulator. As the simulation starts, robot motion navigation is tracked on the V-REP simulator and MATLAB GUI simultaneously. The landmarks are pointed with a red cross while the robot was represented with a small triangle. Run time set at 3000 s. Figure 7 shows MATLAB GUI for robot navigation.

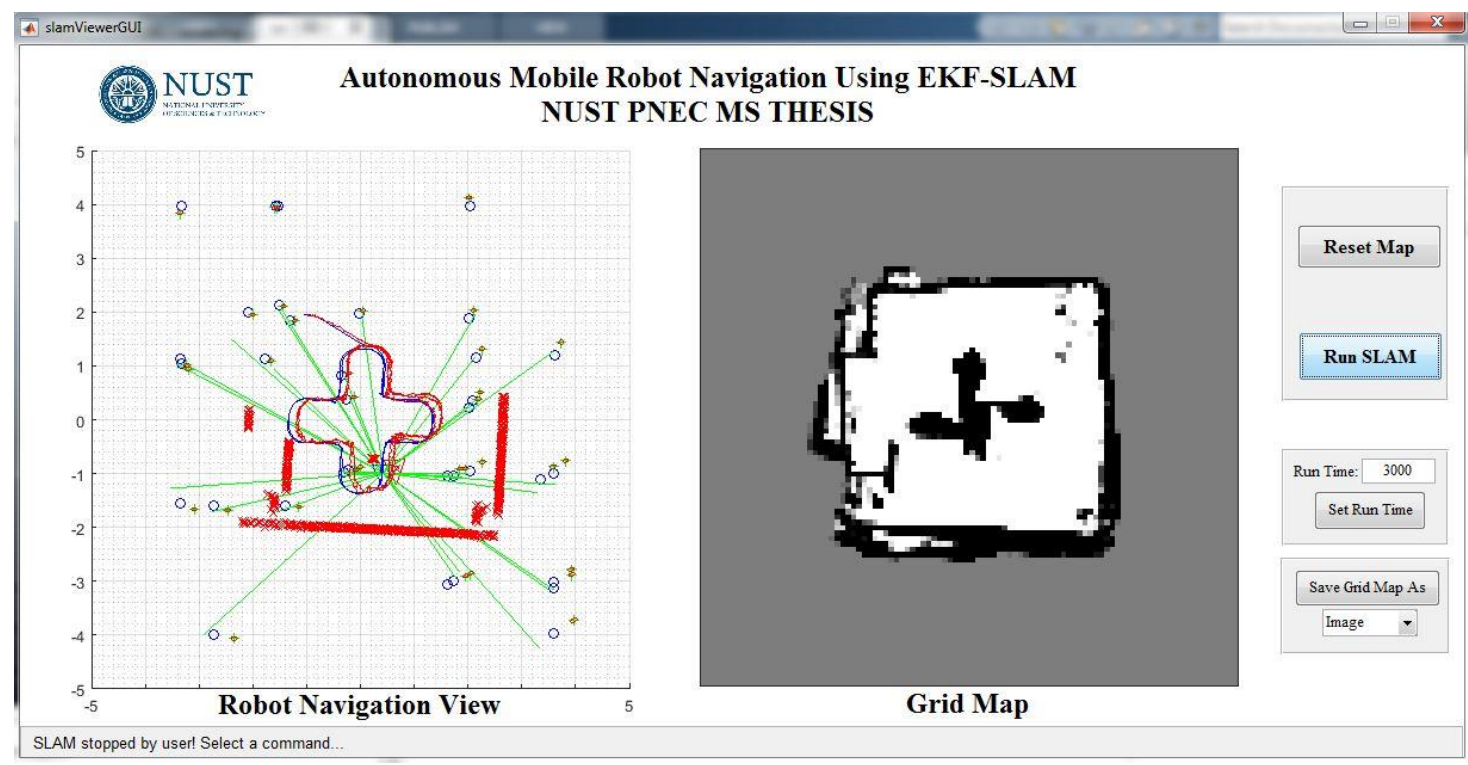

Figure 7. MATLAB GUI for robot navigation 
As the robot moves it find observed landmarks, avoids obstacles, updates states and covariance matrices based on the current map and observation. If any new landmarks are found, it initializes updated states and covariance. While the robot motion-tracked on a grid map, as shown in Figure 8 and Figure 9.

Grid maps are introduced in 1985 by Moravec and Elf, that represents environment by a grid, assuming robot position is known and occupancy of an individual cell is independent stores the posterior probability that a location or corresponding area is occupied by an obstacle, the larger value represents obstacle marked by black color and the smaller value represents free space marked by white color.

Accurate mapping can be achieved by combining lots of data while each cell in the grid represents a bit of the robot's environment indicate some measure of "obstacle Ness" in each grid cell based on laser sensor readings while the algorithm operates if the sensor data has been obtained directly from a laser scanner and using only the odometry [18]-[20].

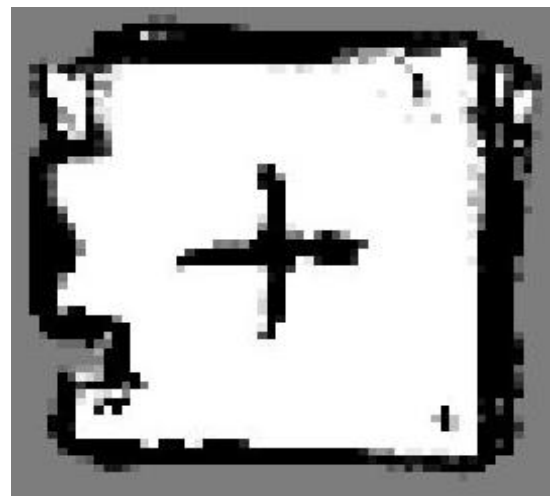

Figure 8. Grid map with static objects

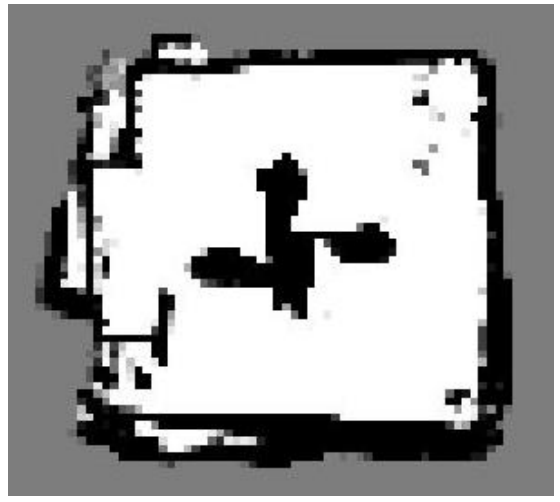

Figure 9. Grid map with dynamic objects

In the robot navigation view, green lines represent lidar sensor exploration, red and blue triangles indicate a mobile robot, blue circles represent landmarks and the Red Cross indicates estimated landmarks, as shown in Figure 10 and Figure 11.

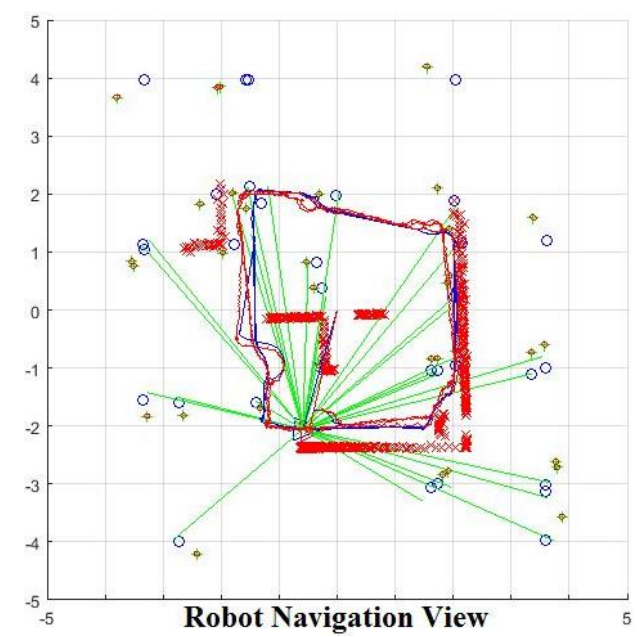

Figure 10. Navigation view with static objects

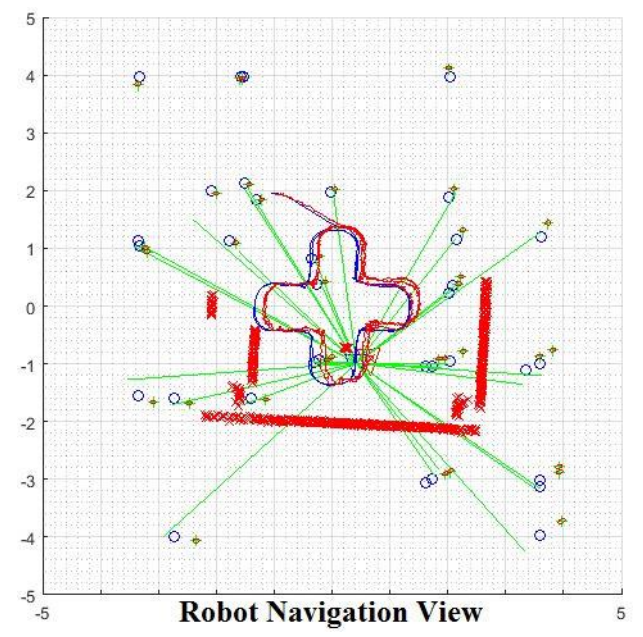

Figure 11. Navigation view with dynamic objects

The performance of the proposed EKF-SLAM algorithm in the indoor environment with static and dynamic objects was observed and compared in terms of the standard deviation of the vehicle heading and it was observed that the uncertainty in position over time is better than usual SLAM results exhibit in Figure 12 and Figure 13. It was observed that the uncertainty in position over time was observed by plotting the standard deviation of position $(\mathrm{m})$ on the $\mathrm{y}$-axis and time(s) on the $\mathrm{x}$-axis, as shown in Table 1. 


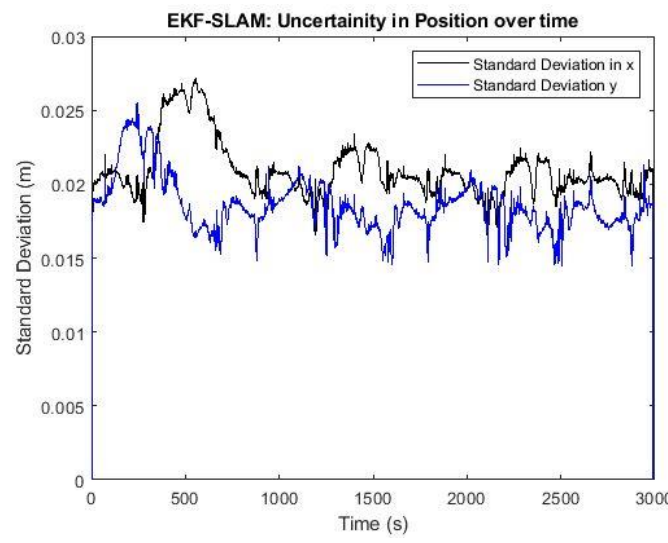

Figure 12. Uncertainty in position over time with static objects

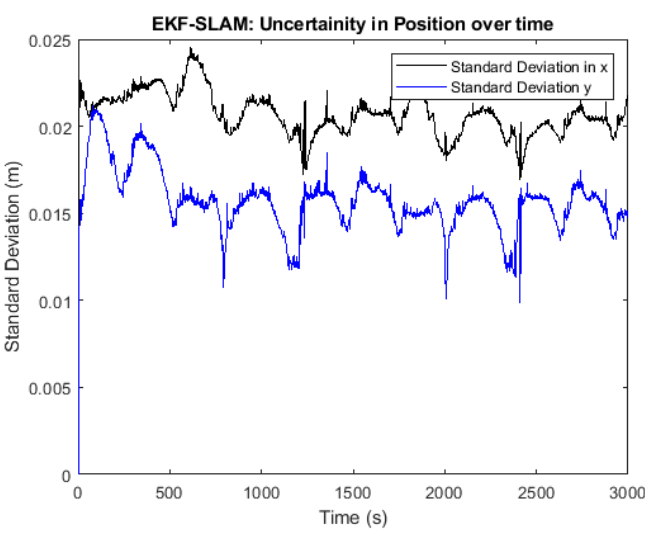

Figure 13. Uncertainty in position over time with dynamic objects

Table 1. Simulated uncertainty in position over time with static and dynamic objects

\begin{tabular}{lcccc}
\hline \multirow{2}{*}{ Uncertainty in position over time } & \multicolumn{2}{c}{ With static objects } & \multicolumn{2}{c}{ With dynamic objects } \\
& Position $(\mathrm{m})$ & Time $(\mathrm{s})$ & Position $(\mathrm{m})$ & Time $(\mathrm{s})$ \\
\hline Maximum standard deviation in x & 0.0278 & 612 & 0.02455 & 615 \\
Minimum standard deviation in x & 0.0175 & 255 & 0.01693 & 2410 \\
Maximum standard deviation in y & 0.0258 & 425 & 0.02145 & 77 \\
Minimum standard deviation in y & 0.0163 & 2917 & 0.00987 & 2412 \\
\hline
\end{tabular}

EKF-SLAM performance and accuracy can also be estimated by plotting errors in position over time. Uncertainty in robot navigation can be computed by considering noise and Jacobians. Figure 14 and Figure 15 represent the error in position over time in the designed indoor environment with static and dynamic objects respectively. It was observed at turning positions inside the room there are spikes after a certain instant of time and more spikes were observed in simulation with dynamic objects at a certain instant of time. In our controlled simulation environment, we have compared the performance of the map joining and the consistency of the mapping algorithm while for some trajectory points discrepancies are observed furthest from the initial location of the vehicle.

When mobile robot heading position is estimated by odometry, Figure 16 and Figure 17 represents scan error over time by plotting position error (m) and angular error (rad) on the y-axis versus time on the $\mathrm{x}$-axis, while odometry error over time represents in Figure 18 and Figure 19. The mobile robot corrects its position by updating landmarks.

Simulation with static objects and dynamic objects results are summarized in Table 2 . It can be analyzed that fewer scan and odometry errors in 3000 seconds simulation time observed with dynamic objects as compared to static objects, although it analyzed that both simulations performed accurate navigation using EKF- SLAM algorithm.

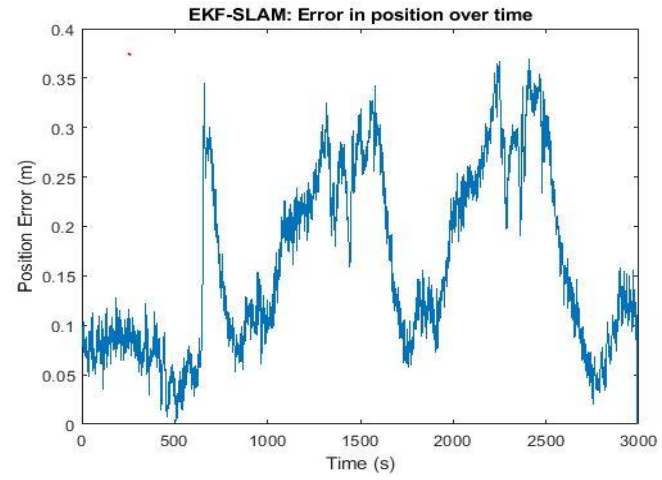

Figure 14. Error in position over time with static

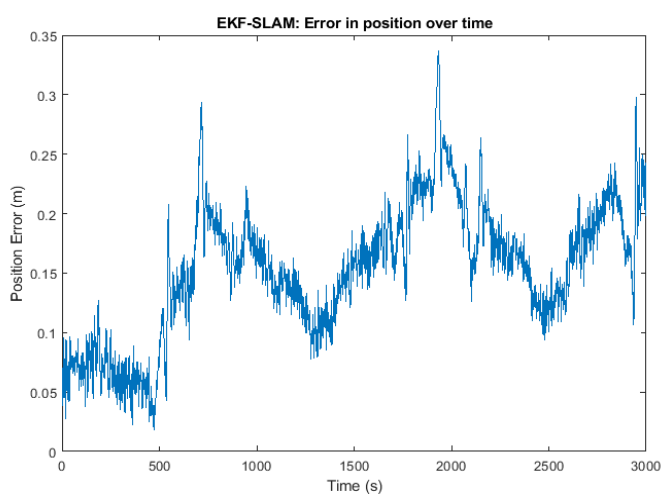

Figure 15. EKF-SLAM-error in position over time 


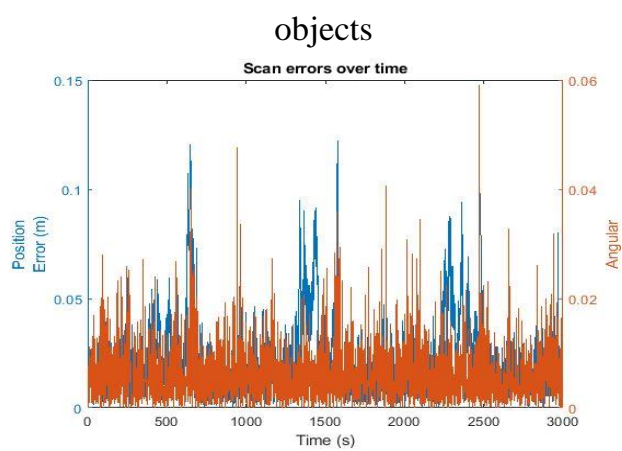

Figure 16. Scan errors over time with static objects

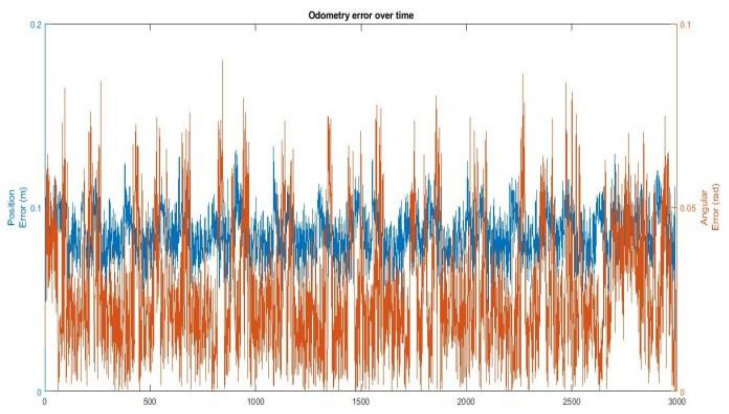

Figure 18. Odometry error over time with static objects

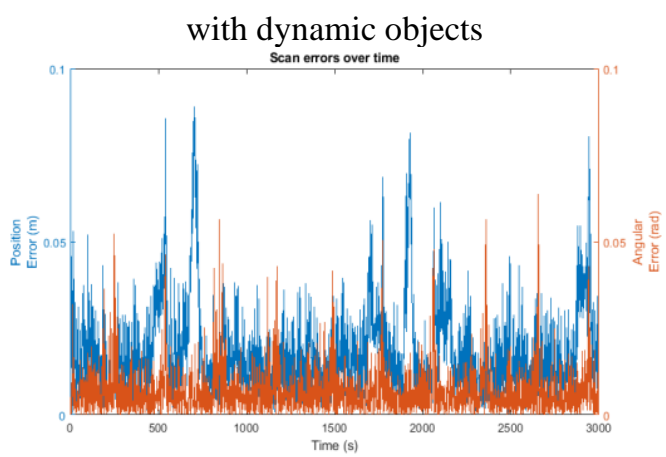

Figure 17. Scan errors over time with dynamic objects

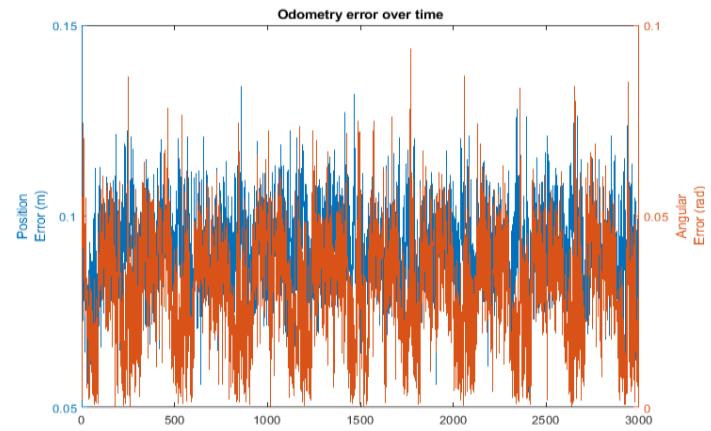

Figure 19. Odometry error over time with dynamic objects

Table 2. Simulation results of EKF-SLAM algorithm

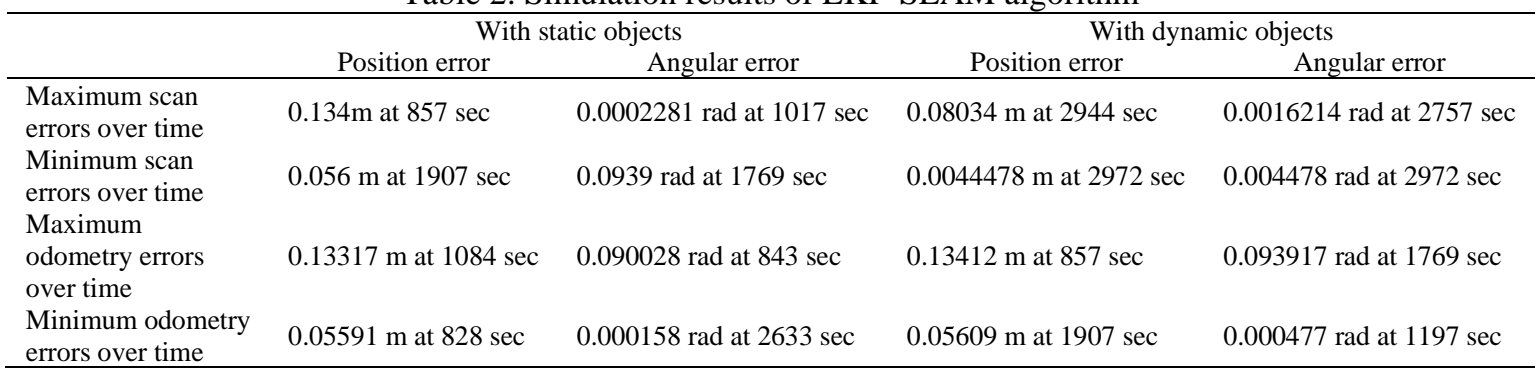

\section{CONCLUSION}

This research represents EKF-SLAM algorithm performance in an indoor environment designed with static and dynamic objects on a V-REP simulator. It was observed and verified with results that autonomous mobile robot navigates using EKF-SLAM algorithm implemented on MATLAB while runtime robot navigation viewed on V-REP which was later linked with MATLAB GUI via remote API commands. The Pioneer 3-DX Mobile robot i.e., equipped with the Lidar and Ultrasonic sensors performed exploration with static and dynamic objects, it was observed through Landmarks measurement that EKF- SLAM corrects odometry error. The simulation verifies that the degradation in odometry error and error in position over time which in results decrease uncertainty in position over time. The robot defines its path by avoiding obstacles and update landmarks if any new landmarks are found during the scan. EKF-SLAM performance observed that the robot performs navigation with less position error, scan error, and odometry error in the indoor environment with static objects as compared to dynamic objects. The suggested EKF- SLAM solves the online SLAM problem by using a linearized Gaussian probability distribution method. It was supposed as first probabilistic SLAM algorithm. Data association is one of the challenging problems in navigation in which association between measurements and features is unknown that is solved using EKF- SLAM.

Future work is to estimate algorithm performance for a longer period with reducing computational complexity, through experiments and compare its result with current research findings. However, EKF-

Realtime autonomous navigation in V-Rep based static and dynamic environment ... (Umme Hani) 
SLAM with consistent information for a longer period is still recommended for navigation for its stable results in complex environments and accurate performance.

\section{ACKNOWLEDGEMENTS}

This research is supported by the Department of Electrical Engineering of Pakistan Navy Engineering College of National University of Sciences and Technology.

\section{REFERENCES}

[1] G. P. Roston and E. P. Krotkov, "Dead Reckoning Navigation For Walking Robots," in Proceedings of the IEEE/RSJ International Conference on Intelligent Robots and Systems, 1992, vol. 1, pp. 607-612, doi: 10.1109/IROS.1992.587401.

[2] J. Park, S. Lee, and J. Park, "Correction Robot pose for SLAM based on Extended Kalman Filter in a Rough Surface Environment," International Journal of Advanced Robotic Systems, vol. 6, no. 2, p. 18, Jun. 2009, doi: $10.5772 / 6789$.

[3] J. Sol'a, Simulataneous localization and mapping with the extended Kalman filter "A very quick guide... with Matlab code!" 2014.

[4] S. Anderson and T. D. Barfoot, "Towards relative continuous-time SLAM," in 2013 IEEE International Conference on Robotics and Automation, May 2013, pp. 1033-1040, doi: 10.1109/ICRA.2013.6630700.

[5] L. Teslić, I. Škrjanc, and G. Klančar, "EKF-based localization of a wheeled mobile robot in structured environments," Journal of Intelligent and Robotic Systems: Theory and Applications, vol. 62, no. 2, pp. 187-203, May 2011, doi: 10.1007/s10846-010-9441-8.

[6] Y. Huang, Y. Zhang, B. Xu, Z. Wu, and J. A. Chambers, "A New Adaptive Extended Kalman Filter for Cooperative Localization," IEEE Transactions on Aerospace and Electronic Systems, vol. 54, no. 1, pp. 353-368, Feb. 2018, doi: 10.1109/TAES.2017.2756763.

[7] G. Reina and A. Messina, "Vehicle dynamics estimation via augmented Extended Kalman Filtering," Measurement, vol. 133, pp. 383-395, Feb. 2019, doi: 10.1016/J.MEASUREMENT.2018.10.030.

[8] A. Kudriashov, T. Buratowski, and M. Giergiel, "Robot's pose estimation in environment exploration process with SLAM and laser techniques," Naukovi Notatki, no. 58, pp. 204-212, 2017.

[9] C. P. Connette, O. Meister, M. Hagele, and G. F. Trommer, "Decomposition of line segments into corner and statistical grown line features in an EKF-SLAM framework," in 2007 IEEE/RSJ International Conference on Intelligent Robots and Systems, Oct. 2007, pp. 3884-3891, doi: 10.1109/IROS.2007.4399404.

[10] S. A. S. Mohamed, M.-H. Haghbayan, T. Westerlund, J. Heikkonen, H. Tenhunen, and J. Plosila, "A Survey on Odometry for Autonomous Navigation Systems," IEEE Access, vol. 7, pp. 97466-97486, 2019, doi: 10.1109/ACCESS.2019.2929133.

[11] Z. Jiang, W. Zhou, H. Li, Y. Mo, W. Ni, and Q. Huang, "A New Kind of Accurate Calibration Method for Robotic Kinematic Parameters Based on the Extended Kalman and Particle Filter Algorithm," IEEE Transactions on Industrial Electronics, vol. 65, no. 4, pp. 3337-3345, Apr. 2018, doi: 10.1109/TIE.2017.2748058.

[12] D. Jung, J. Seong, C. bae Moon, J. Jin, and W. Chung, "Accurate calibration of systematic errors for car-like mobile robots using experimental orientation errors," International Journal of Precision Engineering and Manufacturing, vol. 17, no. 9, pp. 1113-1119, Sep. 2016, doi: 10.1007/s12541-016-0135-4.

[13] F. Jesus and R. Ventura, "Simultaneous localization and mapping for tracked wheel robots combining monocular and stereo vision," Journal of Automation Mobile Robotics and Intelligent Systems, vol. 7, no. 1, pp. 21-27, 2013, Accessed: Oct. 08, 2021. [Online]. Available: https://yadda.icm.edu.pl/baztech/element/bwmeta1.element.baztech85c1da02-8492-4dbd-a0af-e88467ab6fb3.

[14] D. Alejo, F. Caballero, and L. Merino, "A Robust Localization System for Inspection Robots in Sewer Networks," Sensors, vol. 19, no. 22, p. 4946, Nov. 2019, doi: 10.3390/s19224946.

[15] P. Skrzypczynski, "Laser scan matching for self-localization of a walking robot in man-made environments," Industrial Robot: An International Journal, vol. 39, no. 3, pp. 242-250, Apr. 2012, doi: 10.1108/01439911211217062.

[16] G. Kosuru, S. Pedduri, and K. M. Krishna, "Data association using empty convex polygonal regions in EKFSLAM," in 2010 IEEE International Conference on Robotics and Biomimetics, Dec. 2010, pp. 810-815, doi: 10.1109/ROBIO.2010.5723430.

[17] L. D'Alfonso, A. Griffo, P. Muraca, and P. Pugliese, "A SLAM algorithm for indoor mobile robot localization using an Extended Kalman filter and a segment based environment mapping," in 2013 16th International Conference on Advanced Robotics (ICAR), Nov. 2013, pp. 1-6, doi: 10.1109/ICAR.2013.6766461.

[18] F. F. Yadkuri and M. J. Khosrowjerdi, "Methods for Improving the Linearization Problem of Extended Kalman Filter," Journal of Intelligent \& Robotic Systems, vol. 78, no. 3-4, pp. 485-497, Jun. 2015, doi: 10.1007/s10846014-0089-7.

[19] J. Zhang and S. Singh, "Low-drift and real-time lidar odometry and mapping," Autonomous Robots, vol. 41, no. 2, pp. 401-416, Feb. 2017, doi: 10.1007/s10514-016-9548-2.

[20] T. Buratowski, B. Dabrowski, T. Uhl, and M. banaszkiewicz, "The Precise Odometry Navigation for the Group of Robots," Schedae Informaticae, vol. 19, no. 1, pp. 99-111, Dec. 2010, doi: 10.2478/v10149-011-0006-9. 


\section{BIOGRAPHIES OF AUTHORS}
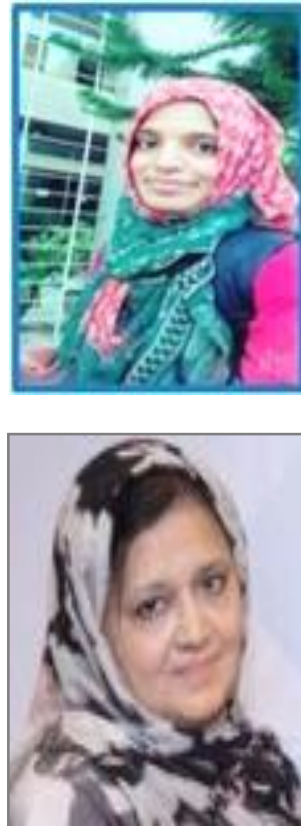

Umme Hani graduated from NED University of Engineering \& Technology, Pakistan, in March 2017 with a Bachelor's of Engineering in Electronics Engineering. She has a background in Industrial Automation, Artificial Intelligence and Machine Vision based systems designed for Pharmaceutical Industries and holds keen interests in the area of Controls Systems. She is currently enrolled in MS Electrical Control System Engineering at National University of Sciences \& Technology, Pakistan. Her research interests include Instrumentation, Industrial Automation, Machine Vision, Control, Robotics and Computer Vision.

Lubna Moin received her Ph.D. in Electrical Engineering from National University of Sciences \& Technology in 2017. She is currently designated as an Assistant Professor in the Department of Electronics \& Power Engineering at National University of Sciences \& Technology, Pakistan. She has published around 20 research articles in leading journals and conference proceedings. She is also a member of IEEE USA and IIIS USA. Her research interests include Modern Control System, Industrial Controls, Machine Learning, Advanced Electronics and Biomedical Engineering. 